\title{
On the connection between Kolmogorov microscales and friction in pipe flows of viscoplastic fluids
}

\author{
H.R. Anbarlooei ${ }^{\text {a }}$, D.O.A. Cruz $^{\text {a }}$, F. Ramos ${ }^{\text {b,* }}$, C.M.M. Santos ${ }^{\text {a }}$, A.P. Silva Freire ${ }^{\text {a }}$ \\ a Mechanical Engineering Program, Federal University of Rio de Janeiro (COPPE/UFRJ), Brazil \\ b Department of Applied Mathematics, Institute of Mathematics, Federal University of Rio de Janeiro, Brazil
}

\section{H I G H L I G H T S}

- New friction factor for viscoplastic fluids with good agreement with experiments.

- Derivation of Kolmogorov microscales for viscoplastic fluids.

- The role of appropriate Kolmogorov microscales for DNS simulations is discussed.

- Limiting shear-thinning behavior similar to Virk's asymptote.

\section{A R T I C L E I N F O}

\section{Article history:}

Available online 5 December 2017

\section{Keywords:}

Kolmogorov microscales

Non-Newtonian fluids

Viscoplastic

Turbulent friction factor

\begin{abstract}
A B S T R A C T
The present work extends Kolmogorov's micro-scales to a large family of viscoplastic fluids. The new micro-scales, combined with Gioia and Chakaborty's (2006) friction phenomenology theory, lead to a unified framework for the description of the friction coefficient in turbulent flows. A resulting Blasiustype friction equation is tested against some available experimental data and shows good agreement over a significant range of Hedstrom and Reynolds numbers. The work also comments on the role of the new expression as a possible benchmark test for the convergence of DNS simulations. The formula also provides limits for the maximum drag reduction of viscoplastic flows.
\end{abstract}

(C) 2017 Elsevier B.V. All rights reserved.

\section{Introduction}

The description of complex fluids and their rheological behavior in a simple and universal mathematical framework is an elusive task. The classical attempt at classifying the known fluids into two different groups, Newtonian or non-Newtonian, has proven to be ineffective, since the term "non-Newtonian" encompasses a wide family of fluids with unrelated physical behavior. Despite this shortcoming, the scientific community has commonly classified complex fluids into three essential groups: purely-viscous, viscoplastic, and viscoelastic (linear and nonlinear). In this work, the scales of Kolmogorov and their relations to the friction coefficient of a large family of turbulent purely-viscous and viscoplastic fluid flows are described. Although these formulations can be seen as the simplest models to describe the behavior of non-Newtonian fluids, they have been commonly used by engineers to describe several processes in the polymer industry, including injection molding, extrusion and pipe flow with heat transfer (see, e.g., [1]).

\footnotetext{
* Corresponding author.

E-mail address: framos@ufrj.br (F. Ramos).
}

For the incompressible flow of a Newtonian fluid, the stress tensor is defined as

$\tau=-\mu\left(\nabla \mathbf{u}+(\nabla \mathbf{u})^{T}\right) \equiv \mu \mathbf{S}$,

where $\mathbf{S}=\nabla \mathbf{u}+(\nabla \mathbf{u})^{T}$ is the rate-of-strain tensor. There are several models concerning viscoplastic fluid flows. A large family of such models is described by a simple generalization of the Newtonian model, by simply replacing the constant viscosity $\mu$, by a shear-rate dependent viscosity, $\mu \equiv \mu(\dot{\gamma})$, where $\dot{\gamma} \equiv \sqrt{2 \mathbf{S}: \mathbf{S}}$ is the second invariant of the rate-of-strain tensor. One important example of this family is the Herschel-Bulkley model, described by

$\tau=\mu(\dot{\gamma}) \mathbf{S}$,

with

$\left\{\begin{array}{l}\mu(\dot{\gamma})=\tau_{y} \dot{\gamma}^{-1}+K \dot{\gamma}^{n-1}, \quad \tau \geq \tau_{y} . \\ \dot{\gamma}=0 \\ \quad \tau<\tau_{y} .\end{array}\right.$

The constant $K$ is the proportionality consistency parameter, and $n$ is the flow index, which measures the degree to which a fluid is shear-thickening $(n>1)$, or shear-thinning $(n<1)$. The parameter $\tau_{y}$ stands for the yield stress, such that for an instantaneous shear stress $\tau<\tau_{y}$, the local deformation rate $\dot{\gamma}$ is null. 
Note that different choices of the above parameters reduce Eq. (1) to other known particular rheologies. For example, as $\tau_{y}=0$, we obtain the purely-viscous model known as power-law fluids. For $n=1$, and $\tau_{y}>0$, the viscoplastic model known as Bingham plastic is obtained. Viscoplastic fluids exhibit a dual response to shear stresses: at low stresses their behavior resembles that of a rigid solid (i.e. $\dot{\gamma}=0$ ); at high stresses they flow like a fluid. This is in contrast to the power-law model, which, despite its variable effective viscosity, does not present a sharp dual behavior at slightly different stresses.

Another well-known viscoplastic model is the Cason model, commonly used to model blood behavior (see [2]). This model can be defined as

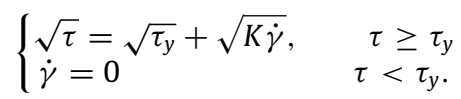

The conjugate effects of shear-thinning and viscoplastic yield stress naturally introduce difficulties in the definition of an appropriate expression for the ratio between inertial and viscous forces, the so-called Reynolds number. For power-law flows, Metzner and Reed [3] proposed a Reynolds number $(R e)$ formulation that is capable of combining all the non-Newtonian parameters into one single expression. In the presence of yielding stresses, the dual nature of the fluid gives rise to an additional non-dimensional parameter, the Hedstrom number $(\mathrm{He})$, which is a measure of the magnitude of the yield stress effects relative to the dynamic Bingham viscosity.

Another difficulty that arises from the complex nature of viscoplastic flows is the description of the near wall viscous effects on the mean axial momentum. The near wall effects are normally encapsulated into a single non-dimensional number, the friction coefficient (or friction factor): $f=\frac{2 \tau_{w}}{\rho U^{2}}$, where $U$ is a characteristic velocity of the flow (usually the mean velocity), $\rho$ is the density of the fluid and $\tau_{w}$ is the wall shear stress, a measure of the force per unit area exerted by the fluid on the wall.

Since the pioneering works of Prandtl and Blasius in the early 1900 s, the physics and engineering communities have established deep connections between the friction factor and other important physical quantities for Newtonian flows, such as the kinetic energy loss and pressure drop in the transportation of fluids in pipes and channels. These relations lead to several formulations of the friction factor as a function of Re. For example, in [4], Blasius established the empirical law $f \sim 1 / R e^{1 / 4}$ to relate the friction factor to the Reynolds number in an interval of validity limited by the onset of turbulence and Re of a few hundred thousand. In [5], Nikuradse extended Blasius' results to included the effects of the roughness on the wall.

Friction formulas have been empirically obtained for several purely-viscous and viscoplastic flows. For example, in [6], Darby and Melson proposed a semi-empirical expression for the Fanning friction factor of fully developed turbulent flow of Bingham fluid, which reads as follows:

$f=10^{a} R_{b}^{-0.193}$,

where $a=-1.47\left[1+0.146 \exp \left(-2.9 \times 10^{-5} \mathrm{He}\right)\right]$, and

$R e_{b}=\frac{\rho U D}{\mu_{b}}, \quad H e=\frac{\rho \tau_{y} D^{2}}{\mu_{b}^{2}}$.

In [7], Dodge and Metzner published an empirical analysis for the friction factor of fully developed turbulent power-law fluid flows based on $R e_{M R}=8 \rho U^{2-n} D^{n} / K(6+2 / n)^{n}$. They obtained the following relation:

$$
\frac{1}{\sqrt{f}}=\frac{4}{n^{0.75}} \log \left(\frac{R e}{f^{\frac{n-2}{2}}}\right)-\frac{0.4}{n^{1 / 2}} \text {. }
$$

For $n=1$, Eq. (5) reduces to the celebrated Prandtl's equation for Newtonian flows. Indeed, the form of DM's equation can be obtained through a Prandtl-Nikuradse scaling of the friction factor, which considers that dissipative mechanisms are the dominant effects in the logarithmic layer, see [8]. In applications, Eq. (5) is the most commonly used friction factor formula for power-law fluids. Many explicit formulas are available in the literature, see, e.g., [9] for a statistical comparison of different formulations. These formulas are mostly empirical models; as such, they lack solid physical foundations.

In [10], Gioia and Chakaborty introduced a phenomenological framework to model Nikuradse's experiments on turbulent friction in rough pipes for Newtonian flows. This work established a phenomenological closure model for the Reynolds' stresses on a wet surface $\mathcal{W}$ in the vicinity of the roughness elements, relating it to the wall shear stress. The main argument in their closure approximation connects the vertical velocity fluctuation component to the Kolmogorov micro-scale velocity.

In $[11,12]$, the present authors have proposed a phenomenological friction coefficient to power-law and Bingham fluid flows, respectively. The theories are valid for smooth pipe and are based on arguments similar to those established in [10]. In those works, the authors have also proposed limits of validity for the friction formulas, which were successfully tested against some available experimental data.

In the present work, the arguments first advanced in [10] are further generalized so as to derive the Kolmogorov micro-scales for general viscoplastic fluid flows. Once the micro-scales are established, the same analysis introduced in [11,12] is used to relate the Kolmogorov micro-scale velocities to the friction formula. The general analysis is validated comparing results obtained through the advanced friction factor formula with some independent experimental data and new DNS data.

We also discuss the limit as the flow index $n$ approaches 0 . As $n$ decreases, the more pronounced is its shear-thinning behavior. We show that as $n$ approaches 0 , the friction formula approaches an asymptote similar to Virk's asymptote which describes the maximum drag reduction for viscoelastic flows.

\section{Extended Kolmogorov theory}

Kolmogorov's K41 theory of fully developed turbulent Newtonian fluid flow was originally formulated on analytical and dimensional grounds and is based on Richardson's energy cascade scenario. The assumption is that energy is injected at large scales, cascades down through intermediate scales, the so called inertial range, and dissipates at the small scales. The cascade hypothesis states that in the inertial range, the average energy flux is constant, independent of the kinematic viscosity, and equals the mean energy dissipation rate, $\epsilon$, see $[8,13]$. Within this phenomenology, the theory is capable of describing how much energy is contained and how much energy is dissipated by eddies of a given size.

Kolmogorov's phenomenology also implies the existence of a transitional scale, $\eta$, called Kolmogorov's dissipative scale or Kolmogorov's micro-scale, between the inertial and dissipative ranges, where both viscous and inertial effects are important, meaning that the eddy turnover time equals the characteristic time that diffusion takes to dissipate energy at the scale $\eta$. Also, the characteristic velocity at this scale, $u_{\eta}$, is known as Kolmogorov's velocity scale.

Experimental [14-16] and numerical evidences [17,18] suggest that Richardson's cascade remains valid for viscoplastic fluids. In this section, we describe the physical arguments that result in Kolmogorov's micro-scales for turbulent viscoplastic fluid flows.

As pointed out in [13], K41 theory is based on three important hypotheses, which must be realized through dimensional arguments and experimental observations. It is clear that the first 
similarity hypothesis (local isotropy of turbulence) is still valid for turbulent flow of viscoplastic fluids. However, the first similarity hypothesis, as stated in [13], should be modified to in every turbulent flow at sufficiently high Reynolds number, the statistics of the small scale motions (micro-scales) have a universal form that is uniquely determined by $\varepsilon$ (mean energy dissipation rate) and the rheology of the fluid. In the Herschel-Bulkley model, the rheology is defined by the set of parameters $\left(\tau_{y}, K, n\right)$ in contrast to the constant viscosity of the established Newtonian model.

The second similarity hypothesis states that in every turbulent flow at sufficiently high Reynolds number, the statistics of the motions of scale $l$ in the range $l_{0} \gg l \gg \eta$ have a universal form that is uniquely determined by $\varepsilon$ and is independent of viscosity. Here $\eta$ represents the Kolmogorov length (micro) scale.

The extension of the second hypothesis for viscoplastic fluid flows is not straightforward, since the inertial range statistics could be, in principle, affected by the formation of micro-plugs in this region. Indeed, the main distinct feature of viscoplastic fluids is the solid-like behavior in unyielded regions of the flow, where the stress is lower than the yielding stress $\left(\tau_{y}\right)$. For example, in the laminar regime, this unyielded region appears as a central plug in the flow.

The central plug is reported to disappear at the end of the laminar to turbulent transition in yielding fluids [14-16,19]. Due to the lack of observation of any micro-plug region in turbulent flow, it is argued that the local stress is always higher than the yielding limit of the fluid in this regime $[16,19]$. A more fundamental explanation is also offered by Frigaard et al. [20] based on the interaction between the flow turbulence and the internal structure of viscoplastic fluids; in this work, the authors argue that the process of crosslink formation and destruction is not instantaneous and yielding fluids show extensive thixotropic properties. The instantaneous zero shear regions do not act like a solid immediately, but, instead, need time to relax to a solid behavior. However, the turbulent time scales are shorter than the relaxation time, so that microplug regions cannot form in turbulent flows [20]. Because of these empirical considerations, we consider that the effective viscosity displayed in the constitutive Eq. (1) simplifies to $\mu(\dot{\gamma})=\tau_{y} \dot{\gamma}^{-1}+$ $K \dot{\gamma}^{n-1}$ in the turbulent regime.

Because of the independence on viscosity, and the hypothesis that the presence of eventual micro-plugs can be neglected, K41's second hypothesis remains unchanged for viscoplastic fluids. As an immediate and crucial consequence, the (-5/3)-law must still hold.

Point-correlations and generalized local Reynolds. We now derive the Kolmogorov micro-scales for general purely viscous fluids. The starting point is the continuity and Navier-Stokes equations (Eq. (6)) with the generalized stress tensor $\left(\tau_{i j}\right)$,

$\frac{\partial U_{i}}{\partial x_{i}}=0 \quad ; \quad \frac{\partial U_{i}}{\partial t}+U_{k} \frac{\partial U_{i}}{x_{k}}=-\frac{1}{\rho} \frac{\partial P}{\partial x_{i}}+\frac{1}{\rho} \frac{\partial \tau_{i k}}{x_{k}}$.

For a general purely viscous fluid, the stress tensor is defined as

$\tau_{i j}=\eta(\dot{\gamma}) S_{i j}$,

where $S_{i j}$ stands for strain tensor, $\mu(\dot{\gamma})=2 \frac{|\tau|(\dot{\gamma})}{\dot{\gamma}}$ is the effective viscosity, and $\dot{\gamma}=\sqrt{2 S: S}$ is the second invariant of strain rate tensor.

Considering the usual Reynolds decomposition of $U, P$ and $\tau$ into $\bar{U}+u^{\prime}, \bar{P}+p^{\prime}$ and $\bar{\tau}+\tau^{\prime}$, one can easily obtain the following equation for the turbulent kinetic energy of homogeneous and isotropic turbulence:

$\rho \frac{d}{d t}\left(K_{t}\right)=-\overline{\tau_{i k}^{\prime} \frac{\partial u_{i}^{\prime}}{\partial x_{k}}}=\rho \varepsilon$
Here, the overline represents time averaging and $K_{t}=\overline{u_{i}^{\prime 2}}$. The righthand side of Eq. (8) is the definition of the dissipation rate $(\varepsilon)$ for purely viscous non-Newtonian fluids. Considering frame invariance, it is easy to show that $\varepsilon \geqslant 0$.

The aim now is to provide an extended version of the local Reynolds number for general viscoplastic fluid flows, based on a spectral analysis of the equations of motion. In the following, the analysis clarifies the terms that remain unchanged, and those which need changes when switching from the analysis of a Newtonian to a non-Newtonian fluid. Once the analysis is established, a locality hypothesis yields an expression for Kolmogorov's microscale. An extension of Lin's equation, see [21], to a viscoplastic fluid follows next.

Multiplying the fluctuating part of the momentum equation at point $A$ with the fluctuating velocity at point $B$ results in

$u_{B j}^{\prime} \frac{\partial u_{A i}^{\prime}}{\partial t}+\frac{\partial u_{A i}^{\prime} u_{A k}^{\prime} u_{B j}^{\prime}}{\partial x_{A k}}=-\frac{1}{\rho} \frac{\partial p_{A}^{\prime} u_{B j}^{\prime}}{\partial x_{A i}}+\frac{1}{\rho} \frac{\partial \tau_{A i, A k}^{\prime} u_{B j}^{\prime}}{\partial x_{A k}}$.

Proceeding similarly for the point $B$, adding the resulting two equations, and also noting that for isotropic turbulence the velocity-pressure correlations at two points are equal to zero, one obtains

$$
\begin{gathered}
\frac{\partial \overline{u_{A i}^{\prime} u_{B j}^{\prime}}}{\partial t}+\frac{\partial}{\partial r_{k}}\left(-\overline{u_{A i}^{\prime} u_{A k}^{\prime} u_{B j}^{\prime}}+\overline{u_{B j}^{\prime} u_{B k}^{\prime} u_{A i}^{\prime}}\right) \\
=+\frac{1}{\rho} \frac{\partial}{\partial r_{k}}\left(-\overline{\tau_{A i, A k}^{\prime} u_{B j}^{\prime}}+\overline{\tau_{B j, B k}^{\prime} u_{A i}^{\prime}}\right),
\end{gathered}
$$

where we have used that $\frac{\partial}{\partial x_{B k}}=\frac{\partial}{\partial r_{k}}$, and $\frac{\partial}{\partial x_{A k}}=-\frac{\partial}{\partial r_{k}}$. To derive Lin's extended equation, one casts Eq. (10) as

$\frac{\partial R_{i j}}{\partial t}=\frac{\partial}{\partial r_{k}}\left(T_{i k, j}+T_{j k, i}\right)-H_{i k j}$,

where $R, T$ and $H$ stand for the double, triple and triple-nonlinear velocity correlations. For the Newtonian case, it is easy to show that

$H_{i k j}=2 v \frac{\partial^{2} R_{i j}}{\partial r_{k} \partial r_{k}}$.

By applying the Fourier transform and making $i=j$, one obtains

$\frac{\partial \Phi_{i i}}{\partial t}=2 k_{k} \Psi_{i k i}-\mathscr{F}\left(H_{i k i}\right)$,

where

$R_{i j}(r, t)=\int \Phi_{i j}(\mathbf{k}, t) e^{i \mathbf{k} . \mathbf{r}} d k \quad ;$

$T_{i k, j}(r, t)=-i \int \Psi_{i j}(\mathbf{k}, t) e^{i \mathbf{k} . \mathbf{r}} d k$,

and $\mathscr{F}(H)$ stands for the Fourier transform of $H$. Using the wellknown relation $\Phi_{i i}(k)=E(k) / 2 \pi k^{2}$, the Lin extended equation results as

$\frac{\partial E(k, t)}{\partial t}=T(k, t)-2 \pi k^{2} \mathscr{F}(H)$

for a general purely viscous non-Newtonian fluid, where $T(k, t)=$ $4 \pi k^{2} k_{k} \Psi_{i k i}(k, t)$. We remark that, unlike the case for Newtonian flows, for general viscoplastic flows, the term $H_{i k j}$ cannot be simplified as in Eq. (12), implying that non-linearity results in a challenging convolution in Fourier space. This fact further complicates the choice of the correct mesh size in DNS simulations of viscoplastic flows.

By integrating Eq. (15), it is possible to show that

$$
\begin{aligned}
\frac{d K_{t}}{d t} & =\int_{0}^{\infty} \frac{1}{2 \pi k^{2}} \frac{\partial E(k, t)}{\partial t} d k \\
& =\int_{0}^{\infty} \frac{1}{2 \pi k^{2}} T(k, t) d k+\int_{0}^{\infty} \mathscr{F}(H) d k=\int_{0}^{\infty} \mathscr{F}(H) d k
\end{aligned}
$$


By comparing Eqs. (16) and (8) one can conclude that $\mathscr{F}(H)=$ $\mathscr{F}(\varepsilon)=\varepsilon_{k}$.

The Kolmogorov micro-scale can be defined as the scale $k_{\eta}$ where both $T\left(k_{\eta}, t\right)$ and $\varepsilon_{k}\left(k_{\eta}, t\right)$ have the same order of magnitude. This also means that the Local Reynolds number at $k_{\eta}$ is approximately 1 . Therefore, at this scale, the following approximation holds:

$\operatorname{Re}_{\eta} \equiv \frac{T\left(k_{\eta}\right)}{2 \pi k^{2} \varepsilon_{k}\left(k_{\eta}\right)} \approx 1$.

Generalized Kolmogorov's micro-scale and localness of interactions. We now obtain an approximation for Kolmogorov's micro-scale by considering a localness hypothesis concerning the interaction of different scales in the inertial range. More specifically, we consider that the energy transfer term represented by $T(k, t)$, after averaging, satisfies $T(k) \sim u_{k}^{3} k^{3}$. We also assume that in this transitional scale, the local energy dissipation rate satisfies $\varepsilon_{k}\left(k_{\eta}\right)=\varepsilon$. From Eq. (17), one, therefore, obtains

$u_{\eta}^{3} k_{\eta} \approx \varepsilon$.

Considering a localness hypothesis for the dissipation term, we obtain by the energy balance, $\rho u_{\eta}^{2} \approx \tau_{k_{\eta}}$, the following equation:

$\rho \varepsilon \approx \rho u_{\eta}^{2} \cdot\left(k_{\eta} u_{\eta}\right) \approx \tau_{k_{\eta}} \cdot\left(k_{\eta} u_{\eta}\right)$.

Combining Eqs. (18) and (19), leads to a general framework to derive Kolmogorov's micro-scales for any such type of rheology.

Herschel-Bulkley. As an example, let us apply this framework to the Herschel-Bulkley model. This leads to

$\rho \varepsilon=\left(\tau_{y}+K\left(k_{\eta} u_{\eta}\right)^{n}\right)\left(k_{\eta} u_{\eta}\right)$.

Using Eq. (18) to eliminate $k_{\eta}$, the Kolmogorov velocity scale can be derived as

$\rho u_{\eta}^{2(n+1)}-\tau_{y} u_{\eta}^{2 n}-K \varepsilon^{n}=0$,

and by considering the length scale $(\eta)$ to be inversely proportional to $k_{\eta}$, the Kolmogorov length scale can be expressed as

$\rho^{3} \varepsilon^{2} \eta^{2(n+1)}-\tau_{y}^{3} \eta^{2 n}-K^{3} \varepsilon^{n}=0$.

Eqs. (21) and (22) are central results in the present work. Their solutions lead, respectively, to Kolmogorov's velocity and length micro-scales for any fluid flow described by the Herschel-Bulkley model.

In particular, if we set $\tau_{y}=0$, we obtain the micro-scales associated to the purely-viscous power-law model, which was first derived in [11]:

$$
\begin{gathered}
\eta \sim\left(\frac{K}{\rho}\right)^{\frac{3}{2(n+1)}} \times \varepsilon^{\frac{n-2}{2(n+1)}} ; \\
u_{\eta} \sim\left(\frac{K}{\rho}\right)^{\frac{1}{2(n+1)}} \times \varepsilon^{\frac{n}{2(n+1)}} .
\end{gathered}
$$

Another interesting example is provided by setting $n=1$. This leads to the micro-scales for the Bingham model, which was developed in [12]:

$$
\begin{aligned}
& \eta \sim\left(\frac{\tau_{y}+\sqrt{\tau_{y}^{2}+4 \rho \varepsilon K}}{2 \rho \varepsilon^{\frac{3}{2}}}\right)^{\frac{3}{2}} \\
& u_{\eta} \sim \sqrt{\frac{\tau_{y}+\sqrt{\tau_{y}^{2}+4 \rho \varepsilon K}}{2 \rho}}
\end{aligned}
$$

Note that by setting $n=1$ and $\tau_{y}=0$, we recover Kolmogorov's micro-scales for Newtonian fluids.

The same procedure can be used for other rheologies. For example, for the Casson model, we obtain

$u_{\eta}=\sqrt{\frac{\tau_{y}}{4 \rho c_{\varepsilon}}}+\sqrt{\frac{\tau_{y}}{4 \rho c_{\varepsilon}}+\sqrt{\frac{K \varepsilon}{\rho c_{\varepsilon}}}}$.

\section{The relation between friction and Kolmogorov scales}

In $[11,12]$, we have proposed new friction expressions for power-law and Bingham fluids, respectively. The formulas were derived through a phenomenological closure model for the Reynolds stress tensor inspired by the work of Gioia and Chakaborty in [10] in the context of Newtonian fluid flows over rough walls. In this section, we extend the previous studies to model the Reynolds stress tensor for general viscoplastic fluid flows bounded by smooth walls.

The phenomenology states that for large Reynolds number, there exists a viscous wet surface $\mathcal{W}$ of constant thickness and parallel to the wall, such that, above it, the horizontal velocity of the flow scales as $\sim U$. In this upper region, the fluid flow carries a high horizontal momentum per unit volume $(\rho U)$. Below $\mathcal{W}$, the velocity of the flow is small, and the fluid has a negligible horizontal momentum per unit volume.

Another important assumption is that over the wet surface $\mathcal{W}$, the stresses are mainly induced by vertical fluctuations of horizontal momentum, so that the net turbulent stresses are dominant over $\mathcal{W}$. Below $\mathcal{W}$, the turbulent stress contribution decays fast, so that in the immediate vicinity of the wall, the main contribution to the stress is due to viscous forces.

The phenomenology proceeds with a pictorial description of eddies that straddle the wet surface $\mathcal{W}$. The eddies transport portions of fluid of high horizontal momentum across $\mathcal{W}$ into the wall direction, thus resulting in portions of fluid with negligible horizontal momentum across $\mathcal{W}$ in the centerline direction. The vortical contribution to the vertical momentum transport across $\mathcal{W}$ is set by $v_{N}$, eddy's velocity normal to $\mathcal{W}$. As in $[11,10]$, the key hypothesis is that $v_{N} \sim u_{\eta}$.

Because viscous stresses are dominated by the inertial vortical fluctuations over $\mathcal{W}$, we consider $\left.\tau\right|_{\mathcal{W}} \sim \rho U u_{\eta}$. This stress is then balanced by the wall shear stress. The wall shear stress satisfies, therefore, the following balance law:

$\tau_{w} \sim \tau_{\mid \mathcal{W}} \sim \rho U u_{\eta}$,

so that, the following friction expression can be advanced:

$f=2 \frac{\tau_{w}}{\rho U^{2}} \sim 2 \frac{\rho U u_{\eta}}{\rho U^{2}}=c_{f} \frac{u_{\eta}}{U}$,

where $c_{f}$ is a constant, that needs to be estimated experimentally.

For statistically stationary turbulence, large eddies must feed energy to the entire inertial range at a rate scaling as $\varepsilon \sim U^{3} / D$. It is possible to combine this with the micro-scales developed in the previous section to find $u_{\eta} / U$. The results are provided in Table 1 , where the generalized Reynolds number $\left(\operatorname{Re}_{G}\right)$ and Hedstrom number $\left(\operatorname{Hed}_{G}\right)$ are defined as

$\operatorname{Re}_{G}=\frac{\rho U^{2-n} D^{n}}{K}$,

$\operatorname{Hed}_{G}=\tau_{y} D^{\frac{-2 n}{n-2}} \rho^{\frac{-n}{n-2}} K^{\frac{2}{n-2}}$

Also in Table 1, Re and Hed are the usual Reynolds and Hedstrom numbers, obtained by letting $n=1$ in the above definitions. 
Table 1

Kolmogorov velocity scales for different viscoplastic models.

\begin{tabular}{lll}
\hline Rheology & Model & $y=u_{\eta} / U$ \\
\hline Newtonian & $\tau=K \dot{\gamma}$ & $y=R e^{\frac{-1}{4}}$ \\
Power-law & $\tau=K \dot{\gamma}^{n}$ & $y=R e_{G}^{\frac{-1}{2(n+1)}}$ \\
Bingham & $\tau=\tau_{y}+K \dot{\gamma}$ & $y=\sqrt{\frac{\frac{H e d}{R e^{2}}+\sqrt{\frac{H e e^{2}}{R e^{4}+\frac{4}{R e}}}}{2}}$ \\
Herschel-Bulkley & $\tau=\tau_{y}+K \dot{\gamma}^{n}$ & $1=y^{2}\left(\operatorname{Re}_{G} y^{2}-H_{e d ~ R e}^{\frac{n}{n-2}}\right)^{\frac{1}{n}}$ \\
Casson & $\sqrt{\tau}=\sqrt{\tau_{y}}+\sqrt{K \dot{\gamma}}$ & $y=\sqrt{\frac{H e d}{4 R e^{2}}}+\sqrt{\frac{H e d}{4 R e^{2}}+\frac{4}{\sqrt{R e}}}$ \\
\hline
\end{tabular}

Table 2

Comparison on the DNS results for turbulent pipe flow with predictions of the theory developed in current work.

\begin{tabular}{|c|c|c|c|c|c|c|}
\hline Rheology & $\operatorname{Re}_{G}$ & $f_{D N S} \times 10^{3}$ & $f_{\text {our }} \times 10^{3}$ & $\Delta f_{\text {our }}(\%)$ & $\Delta f_{e m p}(\%)$ & Ref. \\
\hline Power-Law $(n=0.4)$ & 1400 & 5.94 & 5.944 & 0.07 & 5.0 & [22] \\
\hline Power-Law $(n=0.6)$ & 2615 & 6.70 & 6.755 & 0.80 & 0.9 & [22] \\
\hline Power-Law $(n=0.8)$ & 5125 & 7.34 & 7.346 & 0.08 & 0.6 & [22] \\
\hline Power-Law $(n=1.0)$ & 10320 & 7.87 & 7.873 & 0.38 & 2.0 & [22] \\
\hline Power-Law $(n=1.2)$ & 21245 & 8.21 & 8.206 & 0.04 & 4.0 & [22] \\
\hline $\operatorname{Bingham}\left(\tau_{y} / \tau_{w}=0.1\right)$ & 6058 & 8.932 & 9.029 & 1.0 & 14.0 & - \\
\hline H.B. $\left(n=0.6 ; \tau_{y} / \tau_{w}=0.1\right)$ & 2184 & 6.855 & 7.230 & 5.6 & - & [17] \\
\hline
\end{tabular}

Considering that the averaged energy fed into the system $(\varepsilon)$ should be geometry dependent, $c_{f}$ must be a function of both the rheology and the geometry. However, a comparison with extensive experimental and DNS data reveals that $c_{f}$ depends only on the geometry. Because in the Newtonian limit, the current model recovers the equation of Blasius for pipe flows, $c_{f}$ is considered to be equal to the well-known Blasius coefficient for pipe flows (0.079). For channel flows, the constant $c_{f}$ has been estimated via DNS simulations as discussed in the next section. Therefore, the final form of the general friction equation for pipe flows becomes

$f_{\text {pipe }}=c_{f, \text { pipe }} \frac{u_{\eta}}{U}=0.079\left(\frac{u_{\eta}}{U}\right)$,

$f_{\text {channel }}=c_{f, \text { channel }} \frac{u_{\eta}}{U}=0.059\left(\frac{u_{\eta}}{U}\right)$

where $u_{\eta} / U$ is defined in Table 1 for different rheologies.

\section{Comparison with simulations}

We now compare the proposed friction equation, (Eqs. (30) and (31)), with available DNS results. The determination of the physical properties of any complex fluid is very difficult; in particular, it is not easy to keep results free from contamination. For example, to carry out rheological tests on viscoplastic slurries is naturally cumbersome due to the presence of solid particles in the fluid. For this reason, in many works, only the properties of the solvent are determined. The deviation between the rheological (model) and the real behavior of a fluid is one important source of discrepancy between macro-scale flow predictions and experiments.

To avoid difficulties of the order above reported, the present work resorts to DNS data for theory validation. DNS simulations can be seen as fully controlled non-contaminated experiment. The present comparisons are based on the results of Rudman and Blackburn [17] and Singh et al. [22] for power-law and HerschelBulkley fluids. We also compare the theoretical formulations with our own simulations of Bingham fluid flow in pipes and powerlaw and Herschel-Bulkley fluid flows in channels. The details of the numerical method are described in [18]. In both sets of DNS simulations, an infinite wall-bounded conduit is modeled, with periodic boundary conditions applied stream-wise.

Results for estimation of the friction coefficient for pipe flows through DNS results $\left(f_{D N S}\right)$ and Eq. $(30)\left(f_{\text {our }}\right)$ are shown in Table 2. A comparison with existing empirical friction equations $\left(f_{\text {emp }}\right)$, when permitted, is also presented.

For the power-law fluid, the present theory is compared with the friction equation of Dodge-Metzner, Eq. (5), whereas for the Bingham fluid, the equation of Darby-Melson, Eq. (3), is considered. To the best of our knowledge, no similar friction equation exists for the model of Herschel-Bulkley.

The present theory predicts the power-law results with very high accuracy (less than 1\% error), even for the shear-thickening case, whereas the Dodge-Metzner equation shows errors that can be as high as $5 \%$.

As reported in [18], a decrease in the flow index $(n)$ in the power-law model increases the length of the domain required to obtain statistically converged results. Anbarlooei et al. [18] show that a domain size twice as large as that usually used in DNS simulations of Newtonian flows is sufficient for simulations of power-law flows with indices of $n=0.5$. The current study has found that the domain size must be even larger for the DNS simulation of Bingham and Herschel-Bulkley fluids. Define $\xi=$ $\tau_{y} / \tau_{w}$; the present study has found that by increasing $\xi$, the length of the domain required to obtain statistically accurate results must be greatly increased. (Here, the friction factor is considered the indicator of a statistically converged solution). The length of the domain in the simulations of power-law fluids of [18] is $5 \pi D(D$ is diameter of the pipe), which is twice the size usually used in the simulation of a Newtonian fluid (with $R e_{\tau} \approx 180$ ). It has been here determined that for a Bingham fluid and $\xi=0.1$, the domain length must be much larger $(20 \pi D)$. The results for the Bingham model, presented in Table 2, were obtained with this domain length. Again, predictions of the present theory match well the DNS simulations, while the empirical equations are much less accurate. For a Herschel-Bulkley fluid, the domain size must be even larger, due to the effects of both $\xi$ and $n$. The Herschel-Bulkley fluid results from [17] in Table 2 were obtained in a domain with length of $5 \pi D$. In [17], the authors realized that a larger domain should have been used to capture all energy carrying modes. In any case, the results obtained in the $5 \pi D$ domain still match very well the present theory. Note that no empirical adjustment (fitting) was applied to the present theory.

Although most of the available simulations and experimental data has been obtained for pipe flows, the present theory can be easily extended to other wall-bounded flows, i.e. channel flow. The only difference would be the coefficient $c_{f}$ in Eq. (27), which 
Table 3

Comparison on the DNS results for turbulent channel flow with predictions of the current theory.

\begin{tabular}{lcllll}
\hline Rheology & \multicolumn{1}{c}{$\operatorname{Re}_{G}$} & $f_{\text {DNS }} \times 10^{3}$ & $f_{\text {our }} \times 10^{3}$ & $\Delta f_{\text {our }}(\%)$ & Ref. \\
\hline Newtonian & 2800 & 8.094 & 8.110 & 0.2 & {$[24]$} \\
Newtonian & 6882 & 6.497 & 6.477 & 0.3 & {$[25]$} \\
Newtonian & 10953 & 5.748 & 5.767 & 0.3 & {$[25]$} \\
Newtonian & 18518 & 5.088 & 5.058 & 0.6 & {$[26]$} \\
Newtonian & 43651 & 4.219 & 4.082 & 3.0 & {$[27]$} \\
Power-Law $(n=0.75)$ & 3462 & 5.70 & 5.749 & 0.9 & {$[18]$} \\
Power-Law $(n=0.50)$ & 1862 & 4.82 & 4.795 & 0.6 & {$[18]$} \\
\hline
\end{tabular}

is geometry dependent. In the present work, $c_{f, \text { channel }}$ is obtained by comparing Eq. (27) with the results of the DNS simulation of Newtonian fluid flow in the channel geometry of [23]. These results were here considered due to their higher resolution, large domain size and long statistical averaging. For these results, $c_{f}=0.059$ offers the best fitting to the data.

Table 3 shows a comparison of the predictions of the present theory for channel flow with Newtonian and power-law DNS results. Much in the same way as pipe flow, for the Newtonian case, as the Reynolds number becomes higher than $43651\left(R e_{\tau} \sim 2000\right)$ a discrepancy between the present theory and the DNS results starts to show. The Blasius friction equation (pipe geometry) is known to be valid for $R e<10^{5}$ or, equivalently, $R e_{\tau}<2400$. The mentioned discrepancy for channel flow, can be related to the same phenomenon or just be a consequence of under-resolved simulations at higher Reynolds.

\section{Comparison with experiments}

In this section, we compare the friction expression with experimental results available in the literature. The following comment, however, is in order in relation to some experimental problems frequently noted in the flow of yielding fluids. A direct correlation between pressure and velocity measurements and the rheology of viscoplastic fluids cannot be established even for laminar flows. Most researchers point thixotropy as the cause for this difficulty [14-16,19]. Recently, Dealy [28] argued that pressure is not well-defined for some non-Newtonian fluid flows and questioned the connection between pressure measurements and the state of the stress inside the flow. Considering this fact, in [12] evidence was provided that $\tau_{w}=\Delta P / \Delta L \frac{D}{4}+\tau_{y}$. Considering this argument true, the present friction equation becomes

$f_{P}=0.079\left(\frac{u_{\eta}}{U}\right)-2 \operatorname{Hed}_{G} \operatorname{Re}_{G}^{\frac{-2}{2-n}}$,

where $f_{P}$ stands for the friction factor obtained through pressure measurements. In the rest of this section and in the comparison with the experimental data, Eq. (32) is used.

Fig. 1 shows the results of the comparison of Eq. (32) with the experimental data of $[7,29,30]$ for the power-law model. For power-law fluids a comparison is presented in [11]. The maximum error between predictions and experiments is below 3\%, the same error margin provided by the equation of Dodge and Metzner. However, the present expression, in contrast to the expression of Dodge and Metzner, Eq. (5), reduces to the Blasius equation in the Newtonian limit.

A further comparison is carried out in Fig. 2, for the Bingham model (experiments of [31,32]). Again, predictions furnished by the present theory exhibit a maximum error of $10 \%$; the empirical equation of Darby and Melson, Eq. (3), shows errors as high as $15 \%$.

Fig. 3 contains the experimental results of $[31,19]$ for the Herschel-Bulkley model and predictions provided by the present theory. To demonstrate the flexibility of the friction equation, the experiments are chosen for polymer mixtures ( $0.1 \%$ Carbopol) and solid particle slurries ( $10 \%$ and $14 \%$ Kaolin). As mentioned before,

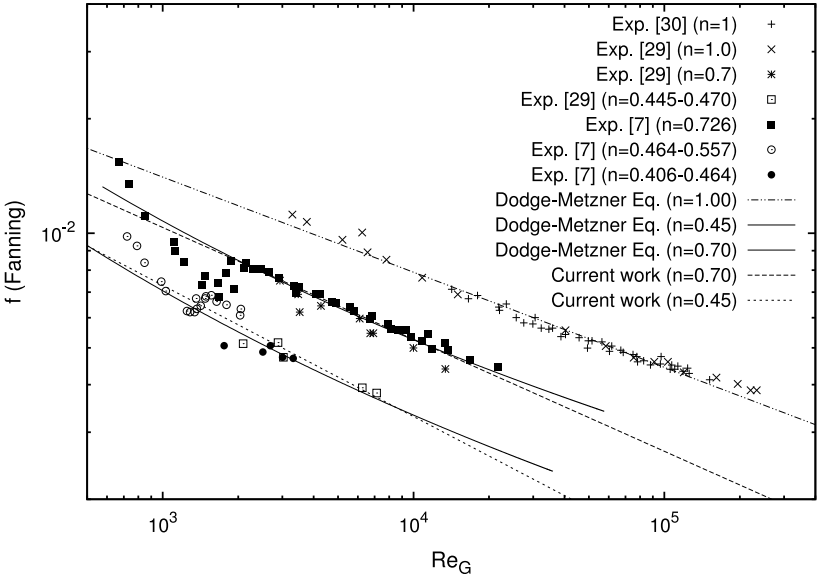

Fig. 1. Comparison between the friction equation developed in the present work with the experimental data of $[7,29,30]$ and Dodge and Metzner's friction equation (Eq. (5)) for power-law fluids.

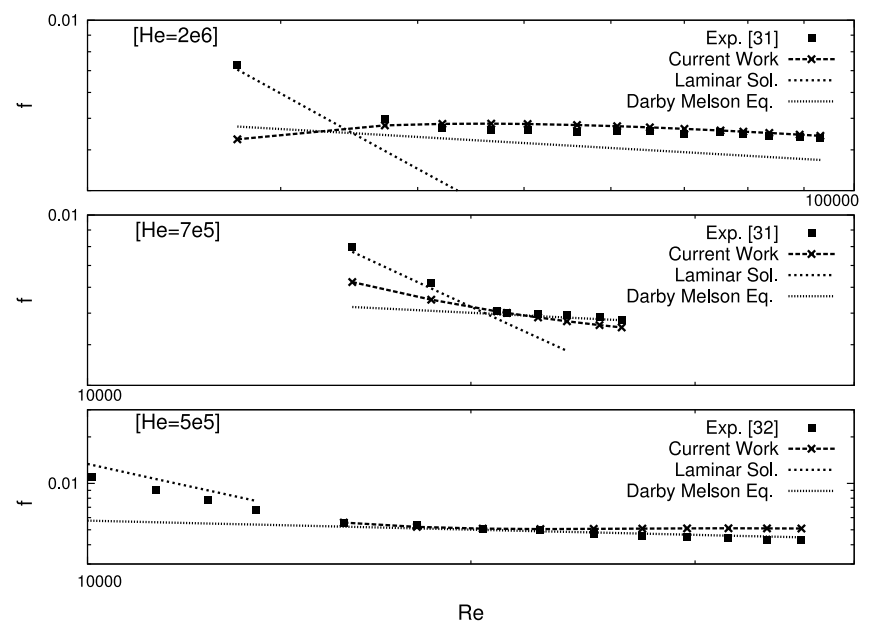

Fig. 2. Comparison between the proposed friction equation and the experimental data of [31,32] and the friction equation of Darby and Melson, Eq. (3), for Bingham fluids.

there exists no empirical friction equation for this model so that it is not possible to compare the accuracy of the present predictions with other equations. A comparison with the experimental results shows that the maximum error is below $6 \%$ for all the cases. A larger set of experimental data [14-16,19,31] has also been used to verify the equation and the maximum error was below $10 \%$.

As we compare the predictions for the Casson model with the available experimental results from [31], we observe an error of the same order as discussed before. Due to lack of enough experimental data on turbulent flow of Casson fluids, this limited comparison is not shown here. 

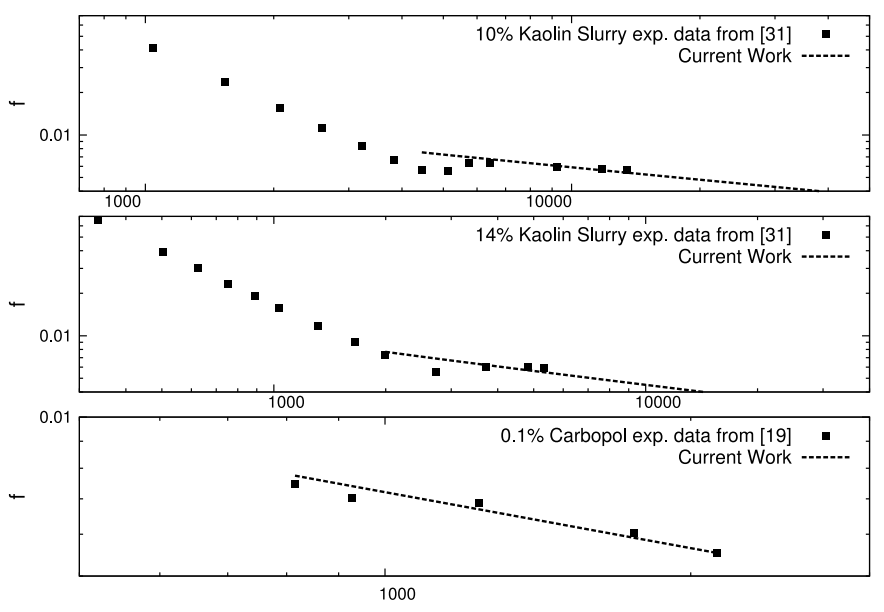

$\operatorname{Re}$

Fig. 3. Comparison between the friction equation introduced in the present work and the experimental data of $[31,19]$ for the Herschel-Bulkley model.

The comparison presented in this section with different rheologies shows that the maximum error of the presently proposed friction equation is under $10 \%$, even for very complex fluids.

\section{Remarks on the geometrical scaling for DNS of complex flows}

An important consequence of defining the appropriate Kolmogorov scales for a given rheology concerns the choice of the appropriate geometrical parameters for the mesh generation of Direct Numerical Simulations of complex fluid flows. Indeed, without any estimate for the Kolmogorov length scaling, any choice for the computational grid spacing is questionable. A review of previous DNS simulations of non-Newtonian fluids, e.g. [17,18,22], shows that grid spacing is obtained through a simple check of grid independence for Newtonian fluids. Without any previous knowledge on the appropriate order of magnitude of the relevant length scales, this process may converge to a local extremum of the turbulence statistics. Within the present framework, the order of grid spacing (length scale) is provided.

The stream-wise domain length plays an important role in the DNS simulation of turbulent flow in pipes (and channels). The effect of the large scale structures is significant in turbulent shear flows, since they contribute notably to the momentum transport processes. An appropriate stream-wise domain length is required to resolve these structures and also to converge to the correct turbulence statistics.

This issue has been studied for Newtonian simulations [33]. For the power-law fluids, it is known that by decreasing the flow index $(n)$ the domain length must increase [17,18,22]. In Fig. 4, the streamwise velocity contours from DNS simulation of power-law fluids are shown for a wall distance of about $y^{+} \sim 10$. Here, the flow indices are 2.0, 1.0, 0.75 and 0.5 from top to bottom. This figure gives a good indication of the streamwise structures that appear in pipe flow. A decrease in $n$ elongates the structures (in the streamwise direction) so that a large domain is required for a correct representation. The same behavior has been reported in [17]. Here, the size of the structures is connected to the Kolmogorov micro-scales, so that a natural conclusion is that for shear-thickening flow ( $n=1.2$ ), the grid spacing can be shorter. The simulations in [18], using a two-point correlation as an indicator, suggest that to $n=0.5$, domains twice as large as the ones commonly used for Newtonian simulation are sufficient, while for the shear-thickening case a shorter domain suffices.
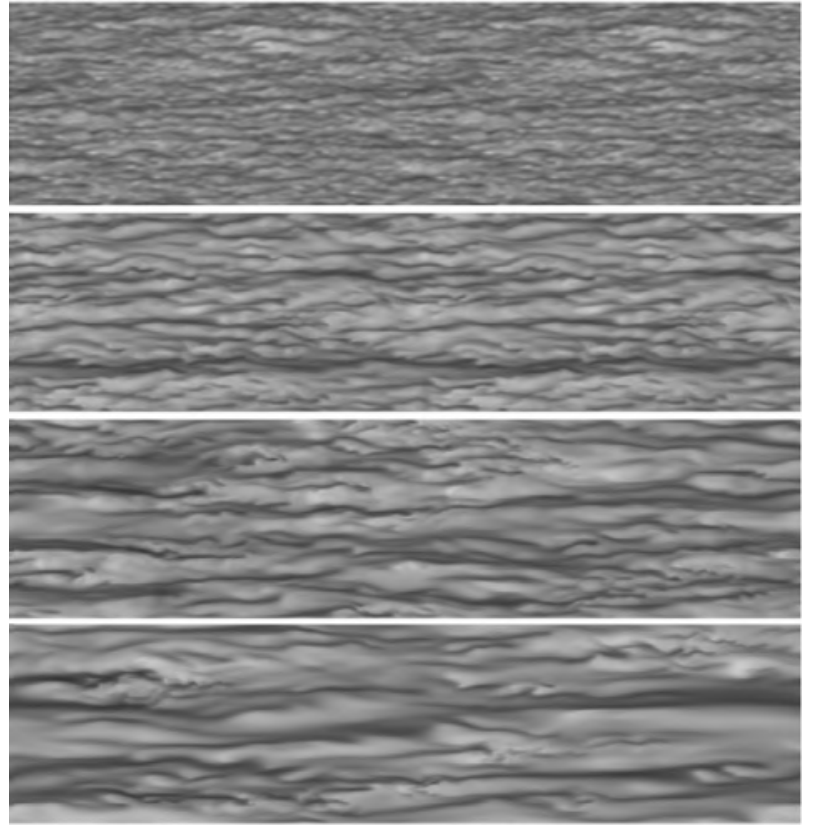

Fig. 4. Contour of axial velocity at $y^{+} \approx 10$ for the power-law model. From top $n$ is 2.0, 1.0, 0.75, and 0.5.
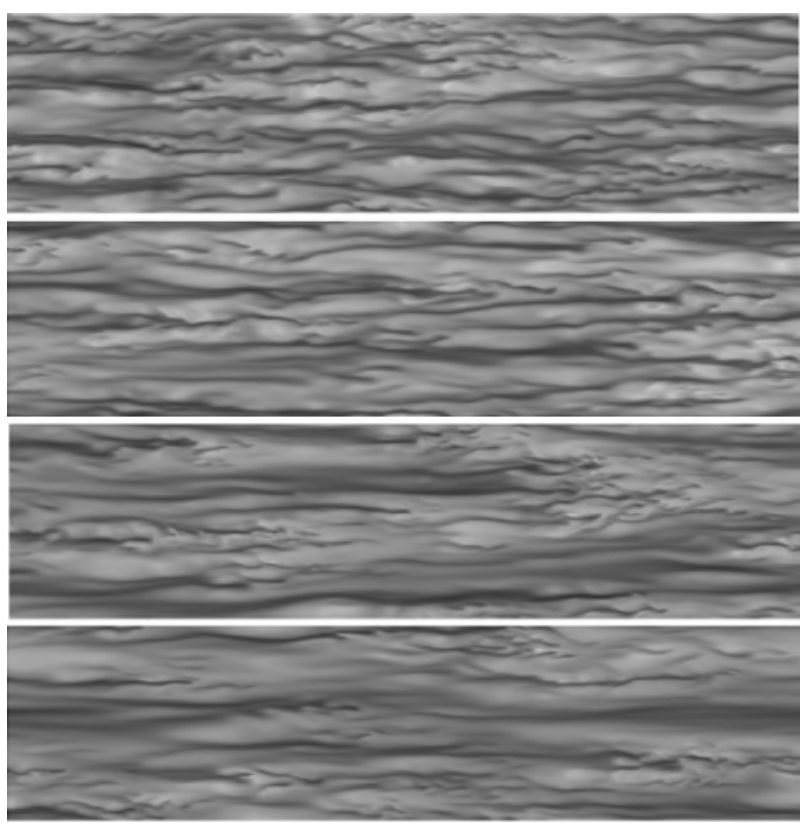

Fig. 5. Contour of axial velocity at $y^{+} \approx 10$ for the Herschel-Bulkley model. From top $\xi=\tau_{y} / \tau_{w}$ is $0.0,0.1,0.2$, and 0.3 .

In Fig. 5, snapshots of the axial velocity contours for simulations of the Bingham model are shown. The same elongated structures in the stream-wise direction are observed, with the increase in $\xi$. The proper size of the grip domain was found to be much larger than the Newtonian counterpart. For example, for the case $\xi=0.1$, it is found that the domain length must be around $20 \pi D$ (for the Newtonian case, the length is $4 \pi D$ ). Such a large domain, prevented us from carrying out simulations with higher $\xi$ or higher Reynolds for these models, due to restricted computational resources.

Because it is expected that, for sufficiently long domains, the velocity fluctuations should be uncorrelated at the center of domain, these correlations are commonly used as a guideline for choosing 
a suitable domain dimension. However, no benchmark result is available on the computational length required to fully isolate the effects of stream-wise periodicity [33]. Based on the present work, it seems that the friction factor can be used as a good candidate for this purpose. This is a potential topic for further research, and we are currently working on this issue.

Another interesting aspect concerns shear-thickening fluid flows. Based on the present theory, the Kolmogorov length scale increases for shear-thickening fluids, in comparison with Newtonian fluids (for a same Reynolds). Also, it is also observed that the stream-wise domain size can be shortened. The implication is that representative DNS simulations of fully turbulent flow could be performed over smaller domains with coarser meshes. The strategy of proposing that a non-Newtonian fluid flow could be simulated through "large-eddy simulation" to approximate some of its features to those of a higher Reynolds number Newtonian flow has been explored by Edriss S. Titi and his collaborators, see, e.g., the references [34,35]

\section{Bounds on the friction coefficient and maximum drag reduction}

An additional important result of the present analysis concerns the bounds of the friction equation. In Fig. 6, the limiting case for the power-law rheology is shown. For this model, the minimum friction is attained as the flow index approaches zero:

$\lim _{n \rightarrow 0} f_{P L}=0.079 R e^{-0.5}$.

This can be considered as the maximum drag reduction due to the shear-thinning effect. Note that the functional form of the limit is very similar to the maximum drag reduction (MDR) in viscoelastic fluids (see, e.g., the asymptote provided by Zakin et al. in [36], which proposes $f=0.315 R e^{-0.55}$ ). The asymptote is also plotted in Fig. 6.

With respect to the upper limit of the friction factor for the power-law fluid, it is easy to see that a constant value $(=0.079)$ is obtained as $n \rightarrow \infty$. For the Bingham and Casson models, the lower limiting is provided by the Newtonian curve; the maximum is obtained for $\xi=1.0$, which is lower than the power-law.

For the Herschel-Bulkley model, the minimum and maximum friction factors coincide with the power-law model. It seems that Eq. (33) represents the maximum drag reduction or minimum friction factor for all flows in the viscoplastic family.

The similarity between Eq. (33) and the empirical MDR asymptotes for viscoelastic fluids leads us to conjecture that there may be a rheology-independent limit for the minimum energy dissipation rate of a turbulent flow. This resembles the famous Onsager's conjecture, which states that there exists an inviscid dissipation mechanism provided by the low-regularity of the "physical solutions" of the incompressible Euler equations, see [37-40]. We are currently investigating those connections.

\section{Conclusion}

In the present work, we have proposed an extended version of Kolmogorov's K41 theory, by providing some suitable adjustments in the similarity hypothesis. To obtain the extended Kolmogorov micro-scales, we have first defined the local Reynolds number as the ratio of the energy transfer term to the dissipation rate term at a fixed wavenumber. Then, by assuming that in the transitional Kolmogorov micro-scale, this local Reynolds number is of order unity, and by a localness hypothesis, Kolmogorov microscales were derived for the non-Newtonian viscoplastic fluids.

We have also presented an important connection between these microscales and the friction in the wall-bounded flows for a

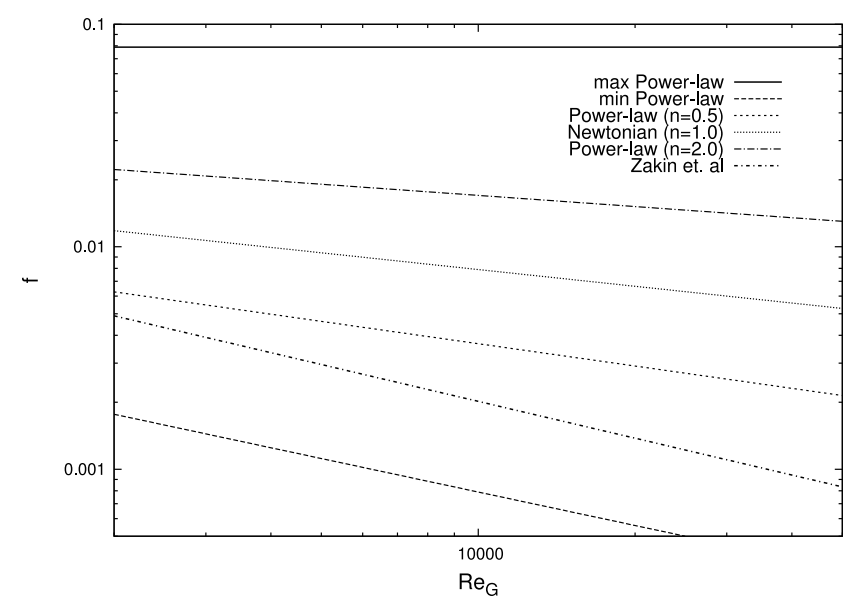

Fig. 6. Minimum and maximum friction factor predicted by the present theory (for power-law model) compared with maximum drag reduction asymptote.

large family of viscoplastic flows, including the Newtonian, powerlaw, Bingham, Herschel-Bulkley and Casson models. This leads us to propose a new friction factor for this family of fluids, which is obtained with no empirical fitting.

We have compared the proposed friction equation with DNS and experimental results. The accuracy of the new friction equation is very good. In particular, the friction equation outperforms the available empirical friction equations. Also, for the HerschelBulkley and Casson models, where no empirical friction equation is available, the present formula results in very accurate predictions.

In $[11,12]$, based on comparisons with wall friction scales, we have established limits of validity for the friction equation, Eq. (30), for power-law and Bingham models, respectively. This was possible due to the explicit expression for the term $u_{\eta} / U$, available for those models. Because for general Herschel-Bulkley models, this ratio can only be obtained through numerical calculations, the limits of validity cannot be explicitly stated. We are currently studying those limits from a numerical point of view.

We have also discussed the importance of the correct definition of the Kolmogorov length scale for purposes of DNS of nonNewtonian fluid flows, which seems to be lacking in the literature. We have also proposed the use of the friction coefficient developed in this work as the benchmark test for the proper setting of the geometrical configuration of the mesh.

The friction expressions presented in this work also establish bounds for the maximum drag reduction for the large family of Herschel-Bulkley fluids, including "drag reducing” fluid flows, obtained by setting $\tau_{y}=0$ and $n<1$. The limiting asymptote resembles that obtained for viscoelastic fluid flows, which is known as Virk's asymptote. To the best of our knowledge, there is currently no available explanation for Virk's asymptote; in particular, there is no theory to directly relate this asymptote to Kolmogorov's cascade structure of turbulent flows. We are currently investigating this connection for different rheologies, including viscoelastic fluid flows. These results will be communicated soon.

\section{Acknowledgments}

A.P.S.F. is grateful to the Brazilian National Research Council (CNPq) for support through the award of a Research Fellowship (Grant No. 305338/2014-5). The work was financially supported by CNPq through Grant No. 477293/2011-5 and by the Rio de Janeiro Research Foundation through Grant Nos. E-26/102.937/2011 and E-26/010.001275/2016 (Pronex). Finally, we would like to thank the anonymous referees for very helpful and insightful comments. 


\section{References}

[1] R. Bird, W. Stewart, E. Lightfoot, Transport Phenomena, Wiley, 2007. A Wiley International edition.

[2] J. Venkatesan, D.S. Sankar, K. Hemalatha, Y. Yatim, Mathematical analysis of casson fluid model for blood rheology in stenosed narrow arteries, J. Appl. Math. (2013). http://dx.doi.org/10.1155/2013/583809.

[3] A.B. Metzner, J.C. Reed, Flow of non-newtonian fluids-correlation of the laminar, transition, and turbulent-flow regions, AIChE J. 1 (4) (1955) 434-440. http://dx.doi.org/10.1002/aic.690010409.

[4] P.R.H. Blasius, Forschungsheft, pp. 1-41.

[5] J. Nikuradse, Forsch. Arb. Ing. Wes. (356).

[6] R. Darby, J. Melson, How to predict the friction factor for flow of bingham plastics, Chem. Eng. 28 (1981) 59-61.

[7] D.W. Dodge, A.B. Metzner, Turbulent flow of non-newtonian systems, AIChE J. 5 (2) (1959) 189-204. http://dx.doi.org/10.1002/aic.690050214.

[8] S.B. Pope, Turbulent Flows, Cambridge University Press, 2000.

[9] P. Gao, J.-j. Zhang, New assessment of friction factor correlations for power law fluids in turbulent pipe flow: A statistical approach, J. Cent. South Univ. Technol. 14 (1) (2007) 77-81. http://dx.doi.org/10.1007/s11771-007-0219-5.

[10] G. Gioia, P. Chakraborty, Turbulent friction in rough pipes and the energy spectrum of the phenomenological theory, Phys. Rev. Lett. 96 (2006) 044502. http://dx.doi.org/10.1103/PhysRevLett.96.044502.

[11] H.R. Anbarlooei, D.O.A. Cruz, F. Ramos, A.P. Silva Freire, Phenomenological blasius-type friction equation for turbulent power-law fluid flows, Phys. Rev. E 92 (2015) 063006. http://dx.doi.org/10.1103/PhysRevE.92.063006.

[12] H.R. Anbarlooei, D.O.A. Cruz, F. Ramos, C.M.M. Santos, A.P. Silva Freire Phenomenological friction equation for turbulent flow of bingham fluids, Phys. Rev. E 96 (2017) 023107. http://dx.doi.org/10.1103/PhysRevE.96.023107.

[13] U. Frisch, Turbulence. The Legacy of A. N. Kolmogorov, Cambridge University Press, 1995

[14] M. Escudier, F. Presti, Pipe flow of a thixotropic liquid, J. Non-Newton. Fluid Mech. 62 (2) (1996) 291-306.

[15] A. Pereira, F. Pinho, Turbulent pipe flow of thixotropic fluids, Int. J. Heat Fluid Flow 23 (1) (2002) 36-51.

[16] J. Peixinho, C. Nouar, C. Desaubry, B. Théron, Laminar transitional and turbulent flow of yield stress fluid in a pipe, J. Non-Newton. Fluid Mech. 128 (2) (2005) 172-184.

[17] M. Rudman, H. Blackburn, Direct numerical simulation of turbulent nonnewtonian flow using a spectral element method, Appl. Math. Model. 30 (11) (2006) 1229-1248, selected papers from the Third International Conference on CFD in the Minerals and Process Industries.

[18] H. Anbarlooei, D. Cruz, A. Silva Freire, The logarithmic solution of purely viscous fluid, in: The 10th International ERCOFTAC Symposium on Engineering Turbulence Modelling and Measurements, 2014.

[19] B. Guzel, T. Burghelea, I.A. Frigaard, D.M. Martinez, Observation of laminarturbulent transition of a yield stress fluid in hagen-poiseuille flow, J. Fluid Mech. 627 (2009) 97-128. http://dx.doi.org/10.1017/S0022112009005813.

[20] I.A. Frigaard, S.D. Howison, I.J. Sobey, On the stability of poiseuille flow of a bingham fluid, J. Fluid Mech. 263 (1994) 133-150. http://dx.doi.org/10.1017/ S0022112094004052.
[21] C. Bailly, G. Comte-Bellot, Turbulence (Experimental Fluid Mechanics), Springer, 2015.

[22] J. Singh, M. Rudman, H.M. Blackburn, The influence of shear-dependent rheology on turbulent pipe flow, J. Fluid Mech. 822 (2017) 848-879. http://dx.doi. org/10.1017/jfm.2017.296.

[23] A.W. Vreman, J.G.M. Kuerten, Comparison of direct numerical simulation databases of turbulent channel flow at re $=180$, Phys. Fluids 26 (1) (2014) 015102. http://dx.doi.org/10.1063/1.4861064.

[24] J. Kim, P. Moin, R. Moser, Turbulence statistics in fully developed channel flow at low reynolds number, J. Fluid Mech. 177 (1987) 133-166. http://dx.doi.org/ 10.1017/S0022112087000892.

[25] R.D. Moser, J. Kim, N.N. Mansour, Direct numerical simulation of turbulent channel flow up to re=590, Phys. Fluids 11 (4) (1999) 943-945. http://dx.doi. org/10.1063/1.869966.

[26] J.C. del Alamo, J. Jimenez, Spectra of the very large anisotropic scales in turbulent channels, Phys. Fluids 15 (6) (2003) L41-L44. http://dx.doi.org/10. $1063 / 1.1570830$.

[27] S. Hoyas, J. Jimenez, Scaling of the velocity fluctuations in turbulent channels up to re $=2003$, Phys. Fluids 18 (1) (2006) 011702. http://dx.doi.org/10.1063/ 1.2162185 .

[28] M. Delay, Misuse of the term pressure in rheology, Rheol. Bull. 77 (2008) $11-13$.

[29] D.C. Bogue, A.B. Metzner, Velocity profiles in turbulent pipe flow. Newtonian and non-newtonian fluids., Ind. Eng. Chem. Fundam. 2 (2) (1963) 143-149. http://dx.doi.org/10.1021/i160006a010.

[30] S.S. Yoo, Heat Transfer and Friction Factors for Non-Newtonian Fluids in Turbulent Flow, University of Illinois at Chicago Circle, US, 1974 (Ph.D. thesis).

[31] D.J. Hallbom, Pipe Flow of Homogeneous Slurry, University of British Columbia, 2008 (Ph.D. thesis).

[32] A. Poloski, H. Adkins, M. Bonebrake, J. Chun, A. Casella, K. Denslow, M. Johnson, M. Luna, P. MacFarlan, J. Tingey, J. Toth, Deposition velocities of non-newtonian slurries in pipelines: Complex simulant testing, Tech. rep., Pacific Northwest National Laboratory, U.S., 2009.

[33] C. Chin, A.S.H. Ooi, I. Marusic, H.M. Blackburn, The influence of pipe length on turbulence statistics computed from direct numerical simulation data, Phys. Fluids 22 (11) (2010) 115107. http://dx.doi.org/10.1063/1.3489528.

[34] B. Levant, F. Ramos, E. Titi, On the statistical properties of the 3d incompressible navier-stokes-voigt model, Commun. Math. Sci. 8 (1) (2010) 277-293.

[35] E. Lunasin, S. Kurien, E.S. Titi, Spectral scaling of $\alpha$-models for two-dimensional turbulence, J. Phys. A 41 (2008)

[36] J.L. Zakin, J. Myska, Z. Chara, New limiting drag reduction and velocity profile asymptotes for nonpolymeric additives systems, AIChE J. 42 (12) (1996) 3544-3546. http://dx.doi.org/10.1002/aic.690421223.

[37] P. Constantin, W. E, E.S. Titi, Onsager's conjecture on the energy conservation for solutions of euler's equation, Comm. Math. Phys. 165 (1) (1994) 207-209.

[38] C. Bardos, E. Titi, Euler equations for incompressible ideal fluids, Russian Math. Surveys 62 (3) (2007).

[39] T. Buckmaster, C.D. Lellis, L.S. Jr., V. Vicol, Onsager's conjecture for admissible weak solutions, arXiv preprint arXiv:1701.08678.

[40] P. Isett, A proof of onsager's conjecture, arXiv preprint arXiv:1608.08301. 